\title{
运用 QC 方法降低转换接头失效率
}

\section{Apply the QC Method to reduce failure rate of X-over}

\author{
马军 ${ }^{1}$ 段延军 $^{1}$ 郑建鲁 $^{1}$ 蔡飞 $^{1}$ \\ Jun $\mathrm{Ma}^{1}$ Yanjun Duan ${ }^{1}$ Jianlu Zheng ${ }^{1}$ Fei Cai ${ }^{1}$ \\ 安东石油技术 (集团) 有限公司 中国·北京 100102 \\ Anton Oilfield Services(Group)Ltd Beijing, 100102, China
}

摘 要: 质量控制是钻井工具服务的关键要素, 本文合理运用 QC 方法对我公司对外租赁的转换接头失效事故进行统计 和分析，找到关键要因并制定相应的解决方案，完善转换接头质量控制措施，有效地降低了转换接头的失效率。

\begin{abstract}
Quality control is the key element of drilling tool service. This paper applies the QC method to analyze the data statistics of X-over failure accident during the service, find out the key causes and formulate corresponding solutions, improve the quality control measures of $\mathrm{X}$-over, and reduce the failure rate of X-over effectively.
\end{abstract}

关键词: QC 方法; 失效率; 质量控制

Key words: QC method; Failure rate; The quality control

DOI: 10.36012 /etr.v2i9.2668

转换接头是石油钻井工具中不可或缺的一部分, 起着 “桥梁” 的作用, 同时它也是整个钻柱最为薄弱的环节之一。 根据统计，我公司平均每年对外提供租赁服务的转换接头高 达 1000 余支次以上, 为了提高工具的稳定性, 我们将 QC 方法运用到转换接头的质量控制中, 降低工具失效率, 以减 少井下事故的发生，提高客户满意度。

\section{1 运用 QC 方法分析问题}

经过统计,2019年我公司对外提供的转换接头约 260 只, 失效共计 3 起。综合失效率为 $1.15 \%$ 。因此, 运用 $\mathrm{QC}$ 方法 对转换接头失效原因进行深人分析，是降低转换接头整体失 效率的关键所在。

\section{2 制定QC 小组活动的目标}

通过对钻工具的分类使用数据统计, 再结合各油田自 有转换接头的使用情况。本次 Q C 小组活动的目标将转换 接头的失效率由 $1.15 \%$ 降低到 $0.5 \%$ 以内，进而全面提高钻 工具的稳定性。

\section{3 运用 QC 方法解决问题}

\section{1 失效原因分析}

结合现场施工的实际情况，小组成员采用树图方式针 对症结原因进行查找和分析, 绘制如图 1 所示的分析图, 共 梳理出 12 个末端因素。

\section{2 要因确认}

在 12 个末端因素中, 液压大钳压力表不准、设计不合理, 狗腿度过大、缩颈严重等 4 个因素通过调查发现，各项均正 常 ; 另外, 地层复杂属于客观因素, 不属于主要分析因素。 因此，剔除 5 个末端因素，对余下 7 个末端因素逐一进行分 析和确认, 确认过程和结果如下。

3.2 .1 末端因素 : 人员未培训、管理漏洞、责任心不强导致 漏检

小组成员调查各井队对操作人员的培训, 发现井队对 员工的培训记录完整，岗位操作人员对上扣扭矩等各项标准 掌握清楚; 核对钻进参数, 确认井队管理过程中各项钻进参 数符合要求, 各项指令参数符合要求; 对井队管理人员及甲 方驻井监督进行核对, 确认井队管理过程符合要求, 未发现

【作者简介】马军 (1974 ), 男, 甘肃合作人，中级工程师，从事钻柱一体化服务及提速提效研究。 


\section{转换接头断裂失效}

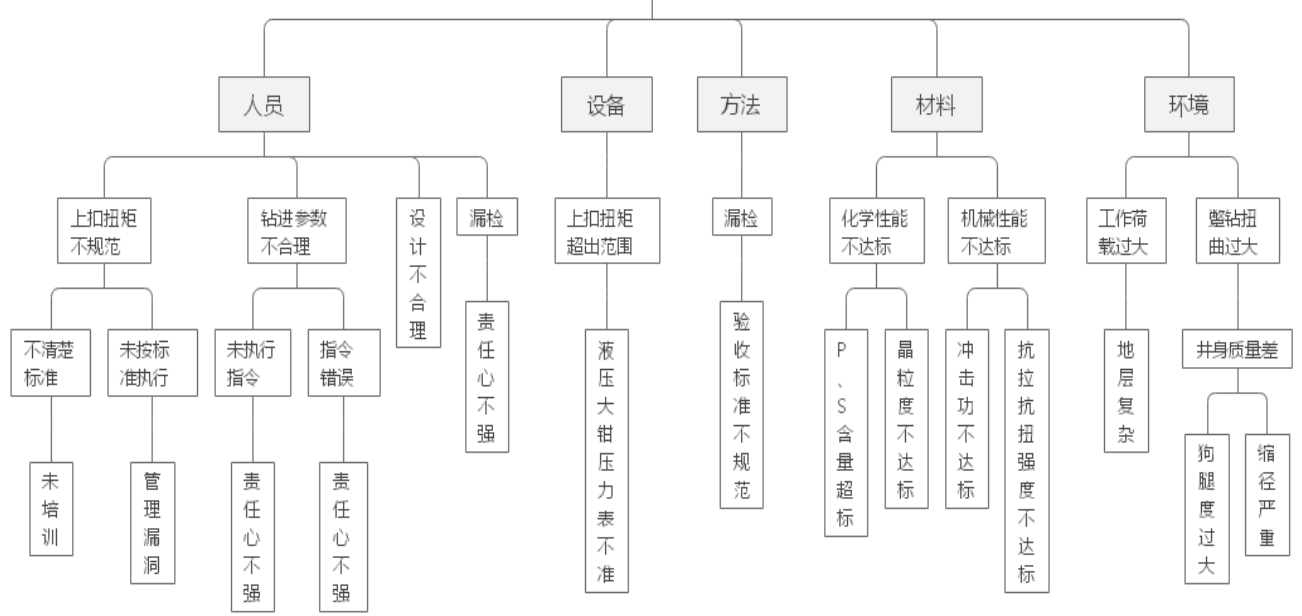

图 1 失效原因分析图

管理漏洞; 对现场 1-3 月份的超声、磁粉检测记录进行检查, 未发现异常，对探伤设备进行检查、测试均符合现场使用要 求，未发现漏检情况。

因此，以上三个末端因素均是非要因。 3.2 .2 末端因素: 化学成分不达标、晶粒度不达标、冲击功 不达标、抗拉强度不达标

小组成员通过对取样送检的结果进行分析, 确认试样 拉伸性能、布氏硬度、非金属夹杂物与晶粒度，以及化学成 分均满足 SY/T 5200-2012 标准和《钻柱转换接头及光坏补 充订货技术条件》; 但夏比冲击试验不满足 SY/T 5200-2012 标准和《钻柱转换接头及光坏补充订货技术条件》, 试验结 果如下：

通过小组成员对末端因素逐一验证，最终确认 “夏比 冲击功不达标” 1 个末端因素为要因。

\section{3 对策制定}

通过进一步的讨论和分析, 确认 “夏比冲击功不达标” 是由到货验收标准不规范、执行力度欠缺和供应商管理不完 善所导致的。为了完成预定目标, 降低转换接头的失效率, QC小组制定对策如下 :

3.3.1 完善到货验收标准和流程, 明确对接头夏比冲击功、 化学成分的验收要求，按标准 $100 \%$ 完成到货验收。具体措 施如下：

严格执行到货产品随机抽样送检标准 ;
加强到货验管理，制定计划，对到货验收标准执行情 况进行监督和检查 ;

完善追责制度，加强管理人员的责任心。

3.3.2 完善对供应商管理规范, 加强对供应商的审查。建全 供应商管理体系，确保执行全面覆盖。具体措施如下：

完善供应商管理体系，制定供应商级别篮选与评定指 标，对不符合要求的供应商进行清退和更换；

加强对供应商原材料采购的审查力度，确保提供的材 质符合要求 ;

补签订货技术协议和商务条款，加强对供应商的管理。 对不符合技术要求的产品予以退货并进行处罚。

\subsection{3 提高工具探伤检测频率，加强质量监督和管理措施 :}

完善探伤检测作业流程, 加强作业人员培训, 提高作 业人员的业务能力;

加强质量监督工作，制定相应的奖惩机制，提高员工 的责任心和积极性 ;

持续开展质量管理评价工作，不断提高公司整体的管 理水平。

\section{4 现场有效开展 QC小组活动}

\section{1 运用 QC方法解决问题的效果}

（1）在对策得到有效实施后，2020 年 1 月至 2020 年 6 月共发井转换接头 570 只，失效 2 起，失效比 $0.35 \%$ 。 
送检试样的夏比冲击试脸结果

\begin{tabular}{|c|c|c|c|c|c|c|c|}
\hline \multicolumn{3}{|c|}{ 试样 } & \multirow{2}{*}{$\begin{array}{l}\text { 溫度 } \\
\left({ }^{\circ} \mathrm{C}\right)\end{array}$} & \multicolumn{4}{|c|}{ 吸收能量 (J) } \\
\hline \multirow{2}{*}{ 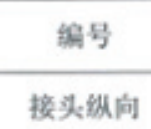 } & $\begin{array}{l}\text { 邫格 } \\
(\mathrm{mm})\end{array}$ & 缺口形状 & & & 4 个1 & & 平均值 \\
\hline & \multirow{4}{*}{$10 \times 10 \times 55$} & \multirow{4}{*}{ V } & \multirow{2}{*}{20} & 19 & 23 & 17 & 20 \\
\hline 接头涉向 & & & & 15 & 17 & 8 & 13 \\
\hline 接头级向 & & & \multirow{2}{*}{-20} & 8 & 8 & 11 & 9 \\
\hline 接头栱向 & & & & 12 & 8 & 10 & 10 \\
\hline \multicolumn{3}{|c|}{$\begin{array}{c}\text { 钻柱转换接头及光俖补充订货技术条件 } \\
\text { (纵向) }\end{array}$} & \multirow{2}{*}{$20 \pm 3$} & \multicolumn{3}{|c|}{$\geqslant 80$} & / \\
\hline \multicolumn{3}{|c|}{$\begin{array}{c}\text { 钻柱转换接头及光俖补允订货技术条件 } \\
\text { (栱向) }\end{array}$} & & \multicolumn{3}{|c|}{$\geqslant 64$} & I \\
\hline \multicolumn{3}{|c|}{$\begin{array}{c}\text { 钻柱转换接头及光俖补允订贷技术条件 } \\
\text { (纵向) }\end{array}$} & \multirow{2}{*}{$-20 \pm 3$} & \multicolumn{3}{|c|}{$\geqslant 65$} & I \\
\hline \multicolumn{3}{|c|}{$\begin{array}{c}\text { 炶柱转换接头及光俖补充订货技术条件 } \\
\text { (頭向) }\end{array}$} & & \multicolumn{3}{|c|}{$\geqslant 52$} & I \\
\hline \multicolumn{3}{|c|}{ SY/T 5200-2012 标准要求 (纵问) } & \multirow{2}{*}{$20 \pm 3$} & \multicolumn{3}{|c|}{$\geqslant 47$} & $\geqslant 54$ \\
\hline \multicolumn{3}{|c|}{ SY/T 5200-2012 标准要求 (横向) } & & \multicolumn{3}{|c|}{$\geqslant 37$} & $\geqslant 43$ \\
\hline
\end{tabular}

(2) 活动前后对比, 转换接头失效比从 $1.15 \%$ 下降到 $0.35 \%$ ，达到小组制定的小于 $0.5 \%$ 的目标，并且持续跟踪 效果良好。

\section{2 如何扎实有效开展 QC小组活动}

（1）制定巩固措施。为了我们的活动成果，持续降低 我公司租赁转换接头的失效率, 进而提高钻工具整体的稳定 性和服务质量。小组根据实际情况, 制定了以下措施。(1)将 活动中采取的改进措施纳人日常管理工作中，并持续改进； (2)将新的管理标准和规范进行全员宣贯和学习;

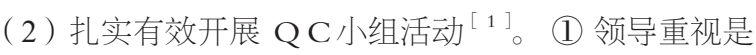
小组活动扎实有效开展的前提，注重小组活动的激励。(2) 强化注册与选题的管理, 使小组活动标准化。 (3) 规范评审
与发布，评审中注重管理技术与专业技术并行，成果层层发 布，逐步提高成果质量。

\section{5 总结与建议}

通过本次活动，不仅成功地实现了既定目标，取得了 较好的经济效益。小组成员分析问题、解决问题的意识得到 了提高，才队合作意识得到了锻炼，也为解决现场实际问题 提供了思路。今后我们将继续分析和研究钻工具服务中出现 的各种问题，不断完善工艺和管理，提高钻工具服务质量。

\section{参考文献}

[1] 秦保华, 李蛟真, 王雪峰, 等. 如何扎实有效开展 Q C 小组 活动 $[\mathrm{J}]$ 。石油工业技术监督，2011，（8）：56 57. 